\title{
Characterization of Neutron Beam Applications
}

\author{
Hirohiko M. Shimizu ${ }^{1,2, *}$ and Katsuya Hirota ${ }^{1,3}$ \\ (on behalf of the Fundamentals Working Group of the Japanese Society for Neutron Science) \\ ${ }^{1}$ Department of Physics, Nagoya University, Furocho, Chikusa, Nagoya 464-8502, Japan \\ ${ }^{2}$ High Energy Accelerator Research Organization (KEK), 1-1 Oho, Tsukuba, Ibaraki 305-0801, Japan \\ ${ }^{3}$ Research Center for Nuclear Physics (RCNP), Osaka University, 10-1 Mihogaoka, Ibaraki, Osaka 567-0047, Japan
}

\begin{abstract}
Analytic framework to quantitatively evaluate the performance of existing neutron scattering instruments would be valuable to find out new possibilities and potentials of slow neutron beams. A conceptual sketch of ongoing effort in Japan is described.
\end{abstract}

\section{Introduction}

Quantitative characterization of the analysis capability of the neutron applications is one of the most fundamental information to overlook the potential of expansion of the neutron science. The common measure of the evaluation has not been sufficiently developed for integrating all potential capability and distributing demands to appropriate facilities. We describe the status of the ongoing effort of the quantitative characterization.

\section{Sketch of a Characterization of the Analysis Capability of a Neutron Scattering Measurement}

We consider the case in which $m$-kinds of observables are obtained in a neutron scattering measurement and put the set of the observables as $\boldsymbol{y}=\left(y_{1}, y_{2}, \ldots, y_{m}\right)$ together with their uncertainties as $\Delta \boldsymbol{y}=\left(\Delta y_{1}, \Delta y_{2}, \ldots, \Delta y_{m}\right)$. When each observable is required to be determined with the accuracy of $\boldsymbol{Y}=\left(Y_{1}, Y_{2}, \ldots, Y_{m}\right)$ all components of

$$
\boldsymbol{\sigma}=\left(\frac{Y_{1}}{\Delta y_{1}}, \frac{Y_{2}}{\Delta y_{2}}, \ldots, \frac{Y_{m}}{\Delta y_{m}}\right)
$$

are required to exceed unity. Here we consider the neutron beam use as the combination of "source", "instrument" and "interpretation" as schematically shown in Fig. 1. We

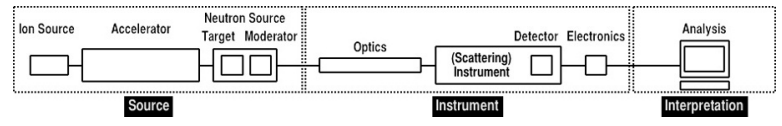

Figure 1. Schematic illustration of the application of the neutron beam driven by accelerators.

use $\Gamma=(\boldsymbol{r}, \boldsymbol{p})$ to represent the phase space of neutrons where $\boldsymbol{r}$ and $\boldsymbol{p}$ are the position and momentum of neutrons. The phase space distribution of neutrons is given as a function of time and can be defined as

$$
\frac{\partial N}{\partial \Gamma}=\frac{\partial^{6} N}{\partial x \partial y \partial z \partial p_{x} \partial p_{y} \partial p_{z}},
$$

where $N$ is the number of neutrons. The phase space distribution evolves over time to that on the moderator surface $\left(\partial N / \partial \Gamma_{0}\right)$, on the entry to the sample $\left(\partial N / \partial \Gamma_{\text {in }}\right)$, on the exit from the sample $\left(\partial N / \partial \Gamma_{\mathrm{ex}}\right)$, which are related as

$$
\begin{array}{cc}
M\left(\Gamma_{0}, \Gamma_{\mathrm{in}}\right) & : \quad \frac{\partial N}{\partial \Gamma_{0}} \rightarrow \frac{\partial N}{\partial \Gamma_{\mathrm{in}}}, \\
M\left(\Gamma_{\mathrm{in}}, \Gamma_{\mathrm{ex}}\right) & : \quad \frac{\partial N}{\partial \Gamma_{\mathrm{in}}} \rightarrow \frac{\partial N}{\partial \Gamma_{\mathrm{ex}}} .
\end{array}
$$

The scattered neutrons are recorded by the detectors as the set of the arrival position and/or timing, which can be described as

$$
M\left(\Gamma_{\mathrm{ex}}, \Gamma_{\mathrm{det}}\right) \quad: \quad \frac{\partial N}{\partial \Gamma_{\mathrm{ex}}} \rightarrow \frac{\partial N}{\partial \Gamma_{\mathrm{det}}} .
$$

Consequently, the raw data of the measurement can be written as

$$
M: \frac{\partial N}{\partial \Gamma_{0}} \rightarrow \frac{\partial N}{\partial \Gamma_{\operatorname{det}}},
$$

with $M \equiv M\left(\Gamma_{\mathrm{ex}}, \Gamma_{\mathrm{det}}\right) M\left(\Gamma_{\mathrm{in}}, \Gamma_{\mathrm{ex}}\right) M\left(\Gamma_{0}, \Gamma_{\mathrm{in}}\right)$. The function of the "instrument" can be considered as the combination of $M\left(\Gamma_{0}, \Gamma_{\text {in }}\right)$ and $M\left(\Gamma_{\text {ex }}, \Gamma_{\text {det }}\right)$. We symbolically use $\mathcal{M}=$ $\left(M\left(\Gamma_{0}, \Gamma_{\mathrm{in}}\right), M\left(\Gamma_{\mathrm{ex}}, \Gamma_{\mathrm{det}}\right)\right)$ to represent the function of the "instrument". The $\mathcal{M}_{\mathfrak{I}}$ is used to represent the $\mathcal{M}$ for a specific "instrument" labeled as $\mathfrak{I}$.

The information of the sample is contained in $M\left(\Gamma_{\mathrm{in}}, \Gamma_{\mathrm{ex}}\right)$ and the observables of interest $\boldsymbol{y}$ is to be numerically derived with their uncertainty $\Delta \boldsymbol{y}$ from the $\partial N / \partial \Gamma_{\text {det }}$. The achievement of the measurement can be quantified in the $\sigma$, which is the comparison of $\Delta y$ and $\boldsymbol{Y}$. We label the analysis method with $\mathcal{I}$ and the $\sigma$ can be expressed as

$$
\sigma=\sigma\left(\Gamma_{0}, \mathcal{M}_{\mathfrak{J}}, \mathcal{I}, T\right)
$$


where $T$ is the measurement time. We consider the case in which the systematic uncertainty is smaller than the requirement $\boldsymbol{Y}$ so that all of the component of $\boldsymbol{\sigma}$ can exceed unity if sufficiently long measurement time is spent. For a specific measurement, denoted by $\vartheta$, we put the minimum measurement time to achieve the requirement as

$$
\tau_{\vartheta}=\tau\left(\Gamma_{0}, \mathcal{M}_{\Im, \vartheta}, \mathcal{I}_{\vartheta}\right),
$$

which measures the performance of the combination of the "source" $\Gamma_{0}$, the "instrument" $\mathcal{M}_{\mathfrak{I}}$, the "interpretation" $\mathcal{I}$ applied for the measurement $\vartheta$.

\section{Optimization of Instrument}

We consider the case where the "instrument" has $n$-kinds of adjustable parameters $\boldsymbol{\alpha}=\left(\alpha_{1}, \alpha_{2}, \ldots, \alpha_{n}\right)$. The optimized condition can be defined as the $\alpha$ to minimize the $\tau_{\vartheta}$, which can be obtained from the $n$-ary simultaneous equation

$$
\frac{\delta \tau_{\vartheta}}{\delta \boldsymbol{\alpha}}=\mathbf{0}
$$

This optimization can be generalized to include all auxiliary conditions of the measurement such as the sample choice, sample temperature, sample pressure, applied magnetic field and so on. Here we put the solution of Eq. 8 as $\left(\boldsymbol{\alpha}_{\vartheta}\right)_{0}$, which is not always achievable. The degree of optimization can be measured in the ratio

$$
\mathcal{S}_{\vartheta}=\frac{\left(\tau_{\vartheta}\right)_{0}}{\tau_{\vartheta}}
$$

which indicates the possibility of further improvement if $\mathcal{S}_{\vartheta}$ is less than unity.

The performance of the "instrument" can be measured by considering the fluence necessary to achieve the requirement of the measurement, which can be written as

$$
F_{\vartheta}=F\left(\Gamma_{0}, \mathcal{M}_{\Im, \vartheta}, \mathcal{I}_{\vartheta}\right) .
$$

The optimization condition of the instrument corresponds to minimize $F_{\vartheta}$.

Each instrument is required to be optimized not only for a single measurement, but also for a set of measurements, denoted by $\mathbb{T}_{\Im}$. Therefore, the instrument optimization can be formulated by the minimization of

$$
F_{\mathbb{T}_{\mathfrak{F}}}=\sum_{\vartheta \in \mathbb{T}_{\mathfrak{S}}} w_{\vartheta} F_{\vartheta}
$$

where $w_{\vartheta}$ is the weight factor for each measurement $\vartheta$, which will be determined on the basis of the subjectively decision. It would be appropriate to redefine the instrument optimization as

$$
\frac{\delta F_{\mathbb{T}_{\mathfrak{F}}}}{\delta \boldsymbol{\alpha}}=\mathbf{0},
$$

and the degree of optimization as

$$
\mathcal{S}_{\mathbb{T}_{\mathfrak{I}}}=\frac{\left(F_{\mathbb{T}_{\mathfrak{I}}}\right)_{0}}{F_{\mathbb{T}_{\mathfrak{I}}}},
$$

where $\left(\boldsymbol{\alpha}_{\mathbb{T}_{\mathfrak{\Im}}}\right)_{0}$ is the solution of Eq. 12 .

\section{Discussion}

The instrument optimization may be generalized to evaluate the degree of optimization of a facility. Most of the neutron facilities have multiple instruments. We put the set of measurements to be carried out in the facility $\mathfrak{R}$ as $\mathbb{T}_{\Re}$. The weighted sum of fluence required to satisfy the requirement of all measurements can be given as

$$
F_{\mathbb{T}_{\Re}}=\sum_{\vartheta \in \mathbb{T}_{\Re}} w_{\vartheta} F_{\vartheta}
$$

However the optimization of Eq. 14 corresponds to the measurement-by-measurement adjustment of instrument parameters in the facility. It would be more practical to minimize the weighted sum of $F_{\mathbb{T}_{\mathfrak{S}}}$ given as

$$
F_{\mathbb{R}}=\sum_{\Im \in \mathbb{R}} w_{\Im} F_{\mathbb{T}_{\mathfrak{S}}}
$$

where $w_{\mathfrak{J}}$ is the weight factor for the instrument $\mathfrak{J}$, which will be determined on the basis of the subjectively decision.

The $F$-value calculated on the basis of common definition will offer a quantitative index to compare instruments or facilities, which will clarify their versatility, uniqueness and significance.

The next step to develop the framework described above is the derivation of the explicit description of the instrument $\mathcal{M}=\left(M\left(\Gamma_{0}, \Gamma_{\mathrm{in}}\right), M\left(\Gamma_{\mathrm{ex}}, \Gamma_{\mathrm{det}}\right)\right)$ for their various usage and also listing of $F$-values of existing instruments and facilities, which is ongoing in the neutron community in Japan. We expect that this framework will clarify the potential of existing methods, their limitations and possible future extensions to activate advanced application of neutron beam. 